\title{
Placenta Accreta: Obstetrician's Nightmare; A Case Series of Seven Patients
}

\author{
Choudhary D, Nigam A, Yadav R, Choudhary S, Raghunandan C \\ Depatment of Obs/ Gyn, Lady Hardinge Medical College and SSK Hospital, New Delhi ,India
}

\begin{abstract}
The incidence of placenta accreta is increasing as a consequence of rising number of cesarean sections and advancing maternal age. A series of seven cases of placenta accreta observed in a short span of four months is being presented. Complication rates in the present series are post partum hemorrhage and major transfusion support (100\%), peripartum hysterectomy $(100 \%)$, ICU admission in four (57\%), fever $(14 \%)$ and bladder injury in one case (14\%).
\end{abstract}

Keywords: Peripartum hysterectomy, placenta accreta, post partum hemorrhage

\section{INTRODUCTION}

The incidence of placenta accreta (PA) is increasing as a consequence of rising number of cesarean sections and advancing maternal age, both being independent risk factors. Third stage of labor in PA is invariably complicated by severe postpartum hemorrhage (PPH) requiring extensive life-saving surgical interventions viz. hysterectomy, ligation of major pelvic vessels and massive blood transfusions. A series of seven cases of PA observed in a short span of four months is being presented.

\section{CASES}

\section{Case 1}

32 years female, G3P1L1A1, at 27+2 weeks gestation with twin pregnancy, was admitted with preterm labor pains. First twin was delivered by vertex and second extracted by internal podalic version, followed by manual removal of placenta in piece-meal. Patient had massive PPH which responded well to balloon tamponade and transfusion support. Balloon tamponade was removed on postpartum day 3 but patient developed fever with leucocytosis in spite of antibiotic cover on day 6. Sonography demonstrated retained bits of placenta. Per speculum examination revealed foul smelling discharge and patient started bleeding heavily after per vaginal examination, and went into shock. On emergency laparotomy, ballooned lower segment with prominent venules were noted and a total hysterectomy performed. (Figure 1a) Histopathological examination revealed placenta accreta with markedly thinned out myometrium and showed areas of infarction and congestion.

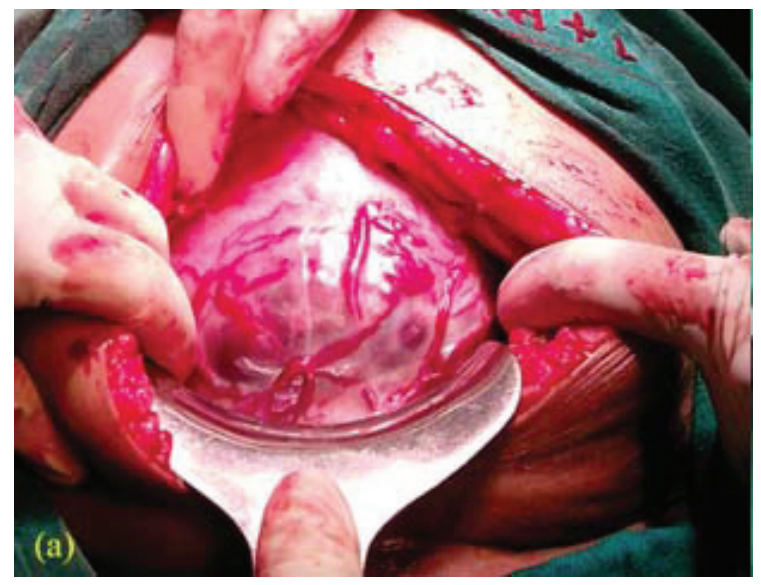

Figure 1(a). Intraoperative photograph showing prominent venules on the surface of uterus.

\section{Case 2}

26 year female, G2P1L1, at $40+2$ weeks gestation with previous lower segment cesarean section (LSCS) presented with ante partum hemorrhage (APH). On emergency

\section{CORRESPONDENCE}

Dr. Deepti Choudhary

Depatment of Obstetrics and Gynaecology

Lady Hardinge Medical College and SSK Hospital

New Delhi, India

Email: dr.deeptichoudhary@gmail.com 
cesarean section, placenta was found to be adherent to the underlying myometrium in lower uterine segment, thus left as such. Uterus was closed with placenta in situ after bilateral uterine artery ligation and internal iliac artery ligation but per operatively profuse fresh bleeding continued vaginally thus hysterectomy (Figure $1 \mathrm{~b}$ ) was performed immediately.

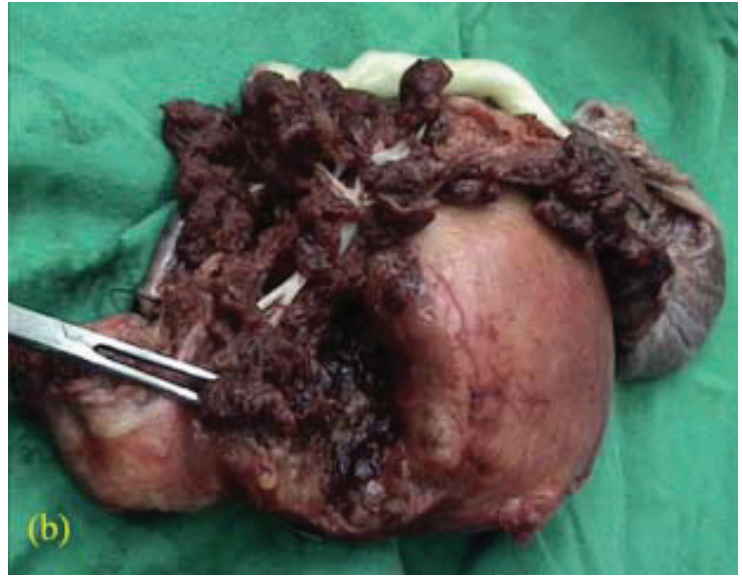

Figure 1(b). Hysterectomy specimen showing placenta invasion in the lower segment

\section{Case 3}

29 years female, G3P2L1, with previous 2 LSCS, at 32+6 weeks gestation, with type IV placenta praevia presented with vaginal bleeding. Per operatively placenta was invading the lower uterine segment. (Figure 1b) Hysterectomy was performed.

\section{Case 4}

25 years female, G3P2L1, 37+5 weeks gestation, with previous 2 LSCS with type IV placenta praevia and suspected PA on sonography was planned for elective cesarean section by upper segment. After baby extraction, patient had intractable PPH and immediate total hysterectomy had to be performed.

\section{Case 5}

On emergency cesarean section in 36 years female, G5P2L2A2, 38+6 weeks gestation with previous 2 LSCS, placenta was found to be morbidly adherent so total hysterectomy was performed.

\section{Case 6}

33 years female with gestational diabetes, previous 2 LSCS, G5P2L2A1 type IV placenta praevia and twin pregnancy with one dead foetus was taken for elective cesarean section at 37 weeks, wherein placenta percreta was present ( figure 1A). Profuse bleeding started after baby extraction by upper segment cesarean section, so hysterectomy had to be performed. Per-operatively, patient sustained bladder injury.

\section{Case 7}

22 years female, G3P2L2, with previous 2 LSCS, with type IV placenta praevia with PA diagnosed on doppler ultrasonography was taken for elective upper segment cesarean section at $37+1$ weeks gestation. Hysterectomy had to be performed in view of intractable PPH.

All the patients required 3-6 units of blood transfusion and component therapy and were discharged in 8 to 10 days postoperatively except one patient who was discharged on $16^{\text {th }}$ day in view of bladder injury. Four patients required post-operative ICU observation. Histopathological examination in all the cases confirmed diagnosis of PA.

\section{COMMENT}

The current incidence of PA is 1 per 2500 deliveries $^{1}$, which has increased to ten folds in the last few decades due to the rising trend of cesarean sections. The reported complications associated with PA are- PPH and massive blood transfusion, peripartum hysterectomy, ICU admission, fever and endometritis. Major risk factors for PA are placenta praevia and previous cesarean section. The complications associated in this study were PPH and major transfusion support (100\%), peripartum hysterectomy (100\%), ICU admission in four (57\%), fever (14\%) and bladder injury in one case (14\%). Similar complication rates as in the present series have been reported. ${ }^{2}$ Three of the seven cases were suspected of PA antenatally; six cases were previous LSCS of which 5 had placenta praevia. Moreover, the remaining case that was managed conservatively had previous history of dilatation and curettage. Therefore, all the cases had a high risk factor, thus emphasizing the need to carefully evaluate the placental implantation site at the time of routine ultrasound and also the need to maintain a high index of suspicion in cases bearing identified high risk factors. Placenta praevia has been reported to be associated with PA in $10 \%$ of cases. $^{3}$

The diagnosis of PA can be made using color flow doppler ultrasound as early as the second trimester. Typical features of a placenta accreta ${ }^{4}$ include loss of normal hypoechoiec boundary between the placenta and urinary bladder or serosa, appearance of placenta being contiguous with the bladder wall, visible sonolucent spaces within the placenta, adjacent to the uterine walls, and persistent blood flow between the basal placenta and myometrium revealed on color doppler.

Placental lacunae and abnormal color doppler imaging patterns are the most helpful sonographic markers for PA. More recently, magnetic resonance imaging has been used for evaluation of PA. It is more reliable in the presence of posterior placenta and for assessing depth of invasion when USG findings are equivocal. ${ }^{5}$ 
Both sonography and MRI have fairly good sensitivity for prenatal diagnosis of PA; however, specificity may not be as good. Diagnosis is often made when there is difficulty in establishing a cleavage plane during removal of the placenta as seen in the three unsuspected cases of emergency LSCS. Manual efforts to remove the adherent placenta result in massive blood loss and even unstable hemodynamic status. Removing the placenta in the hope of avoiding hysterectomy is rarely effective. ${ }^{2}$ Hysterectomy has been traditionally advised for the management of PA, but conservative management comes at the cost of higher rates of maternal sepsis. ${ }^{6,7}$ There is still no consensus about an optimal protocol. Conservative management can be considered in women without massive PPH where fertility needs to be preserved. A conservative strategy leaves the placenta in situ without forced removal during delivery. Conservative management attempted in two of the present cases failed- one case required delayed hysterectomy while the bleeding continued per-vaginally in the other. The latter case emphasizes the need to check for per-vaginal bleeding intraoperatively if placenta is left in situ.

\section{REFERENCES}

1. Morgan M, Atalla R. Mifepristone and the misprostol for the management of placenta accreta- a new alternative approach. BJOG. 2009;116(7):1002-3

2. Eller A, Porter T, Soisson P, Silver R. Optimal management strategies for placenta accreta. BJOG. 2009;116(5):648-54

3. Uszynski W, Uszynski M. Placenta accreta: epidemiology, molecula mechanism (hypothesis) and some clinical remarks. Ginekol Pol. 2004; $75: 971-8$.

4. Comstock $\mathrm{CH}$. Antenatal diagnosis of placenta accreta: a review. Ultrasound. Obstet Gynecol. 2005;26(1):89-96.
It is worth highlighting from these cases that a high index of suspicion in high-risk cases should be kept to increase the chances of antenatal diagnosis. We believe that one should never try to separate the morbidly adherent placenta. If decision to leave the placenta has been taken, careful monitoring for per vaginal bleeding and the maternal sepsis should be done. Decision for hysterectomy should be taken liberally if there is severe $\mathrm{APH} / \mathrm{PPH}$, in order to avoid maternal mortality and morbidity. All the cases with high risk factors and suspicion of PA should be managed where skilled facilities of obstetrician, anesthetist, ICU and blood bank are available.

\section{CONCLUSION}

PA is an obstetric challenge that is being encountered with increasing frequency and is associated with high maternal mortality and morbidity. Clinicians should be aware of the clinical issues, risk factors, and imaging findings associated with PA to facilitate optimal case management. Scheduled cesarean hysterectomy performed under controlled circumstances, without any attempt at removing the placenta, availability of adequate transfusion support significantly reduce maternal morbidity and mortality.

5. Baughman WC, Corteville JE, Shah RR. Placenta accreta: spectrum of US and MR imaging findings. Radiographics. 2008;28:1905-16.

6. Dwyer BK, Belogolovkin V, Tran L, Rao A, Caroll I, Barth R, et al. Prenatal diagnosis of placenta accrete: sonography or magnetic resonance imaging? J Ultrasond Med . 2008;27(9):1275-81.

7. Bennett MJ, Sen RC. Conservative management of placenta praevia percreta: report of two cases and discussion of current management options. Aust N Z J Obstet Gynaecol. 2003;43(3):24951. 